\title{
Interfaces with superroughness
}

\author{
Ning-Ning Pang ${ }^{1}$ and Wen-Jer Tzeng ${ }^{2}$ \\ ${ }^{1}$ Department of Physics, National Taiwan University, Taipei, Taiwan, Republic of China \\ ${ }^{2}$ Department of Physics, Tamkang University, Tamsui, Taipei, Taiwan, Republic of China
}

(Received 11 October 1999; revised manuscript received 16 December 1999)

\begin{abstract}
We undertake an extensive analytical study of the $(1+1)$-dimensional discrete superrough growth processes, which are the growth processes with the global roughness exponent larger than 1 . First, we obtain the exact expressions of the global interfacial width $w(L, t)$, the local interfacial width relative to the substrate orientation $w(l, t)$, and the local interfacial width relative to the local interfacial orientation $w_{n}(l, t)$, in terms of the equal-time height difference correlation functions $G(r, t)$. These relations are exact and can be applied to all the $(1+1)$-dimensional discrete growth processes with periodic boundary conditions. Moreover, we show that the local roughness exponent must be smaller than 1 for the $(1+1)$-dimensional superrough growth processes with $w_{n}(l, t)$ retaining the same anomalous dynamic scaling behaviors as $w(l, t)$; in contrast, the local roughness exponent must be equal to 1 for those with $w_{n}(l, t)$ retrieving the ordinary dynamic scaling behaviors.
\end{abstract}

PACS number(s): 05.40.-a, 47.55.Mh, 64.60.Ht, 68.35.Ct

The kinetic roughening phenomenon of growing interfaces [1-3] has brought about much interest for its widespread applications. Recently, much attention has been focused on the superrough growth processes, which are the growth processes with the global roughness exponent $\chi>1$. Among all the experimentally accessible quantities, one of the most informative quantities is the equal-time height difference correlation function $G(r, t)$. Here, $G(r, t)$ is defined as

$$
G(r, t) \equiv \overline{\left\langle\left(h\left(x_{0}, t\right)-h\left(x_{0}+r, t\right)\right)^{2}\right\rangle_{L}},
$$

with $h(x, t)$ denoting the interface height from a flat substrate at position $x$ and time $t,\langle\cdot\rangle_{L}$ denoting the spatial average over the whole system of size $L$, and the overbar denoting the statistical average, throughout the paper. For various superrough growth processes with either annealed or quenched noises, the equal-time height difference correlation function $G(r, t)$, in the regime where the correlation length $\xi \sim t^{1 / z}$ $\ll L$, has been both numerically [4-7] and experimentally [8-10] observed to display the anomalous dynamic scaling behaviors:

$$
G(r, t)=r^{2 \chi} f\left(r / t^{1 / z}\right)
$$

with the scaling function $f(y)$ obeying

$$
f(y) \sim \begin{cases}y^{-2 \kappa} & \text { for } y \ll 1, \\ y^{-2 \chi} & \text { for } y \gg 1 .\end{cases}
$$

Here, the two independent exponents $\chi$ and $z$ are known as the global roughness exponent and the dynamic exponent, respectively. Note that for the ordinary dynamic scaling behavior [11] displayed by the truly self-affine interfaces, the scaling function goes to a constant quickly in the small $y$ limit. Thus, the appearance of the third independent nonzero exponent $\kappa$, i.e., the anomalous temporal dependence in the intermediate time regime $r \ll t^{1 / z} \ll L$, is the signature of anomalous dynamic scaling behaviors.
For one decade, people have generally believed that the kinetically roughened interfaces are self-affine; namely, the local interfacial width and the equal-time height difference correlation function have the same scaling behavior as the global interfacial width, which has been known to obey the ordinary dynamic scaling ansatz. Therefore, the numerical and experimental establishment of the existence of anomalous dynamic scaling behaviors is an important breakthrough in the study of interfacial kinetic roughening phenomena. Although many numerical works have been done, rigorous analytical treatments are still rare. This motivates us to undertake an extensive analytical treatment on the $(1+1)$-dimensional discrete superrough growth processes with periodic boundary conditions. We first want to obtain the exact expressions of the global interfacial width $w(L, t)$, the local interfacial width relative to the substrate orientation $w(l, t)$, and the local interfacial width relative to the local interfacial orientation $w_{n}(l, t)$, in terms of the equal-time height difference correlation functions $G(r, t)$. Then, we will employ the obtained relations to explore the intriguing interfacial behaviors of the $(1+1)$-dimensional superrough growth processes in the intermediate time regime.

Let us consider a one-dimensional interface represented by a set of height variables $h(x, t)$ on a linear lattice $x$ $=1,2, \ldots, L$ with periodic boundary conditions. The global interfacial width $w(L, t)$ is defined as

$$
w^{2}(L, t) \equiv \overline{\left\langle\left(h(x, t)-\langle h(x, t)\rangle_{L}\right)^{2}\right\rangle_{L}},
$$

which describes the interface height fluctuation relative to the average interface height over the whole system of size $L$. In contrast, the local interfacial width $w(l, t)$ is defined as

$$
w^{2}(l, t) \equiv \overline{\left\langle\left\langle\left(h(x, t)-\langle h(x, t)\rangle_{l}\right)^{2}\right\rangle_{l}\right\rangle_{L}}
$$

with $\langle\cdot\rangle_{l}$ denoting, throughout the paper, the spatial average calculated within a local window of size $l$. The local interfacial width $w(l, t)$, obtained by averaging over many local windows of the same size $l$ along the $x$ axis and then over the 
randomness, describes the interface height fluctuation relative to the average interface height within the local window of size $l$ ( $\ll$ the system size $L$ ). To extract out the effect of local interfacial orientational instability on interfacial roughening, we have proposed [12] a definition of the local interfacial width $w_{n}(l, t)$ as follows:

$$
w_{n}^{2}(l, t) \equiv \overline{\left\langle\left\langle\left(h(x, t)-\widetilde{h}_{l}(x, t)\right)^{2}\right\rangle_{l}\right\rangle_{L}},
$$

which describes the interface height fluctuation relative to the local interfacial orientation within a local window of size $l$ (« the system size $L$ ). Here, $\widetilde{h}_{l}(x, t)$ denotes the heights, measured from the flat substrate, of a straight line segment obtained by least squares fit to the interfacial configuration in the local window of size $l$ at a given time $t$. Quantitatively,

$$
\widetilde{h}_{l}(x, t)=\langle h(x, t)\rangle_{l}+\left(x-\langle x\rangle_{l}\right) s(l, t)
$$

with

$$
s(l, t)=\frac{12}{\left(l^{2}-1\right)}\left\langle\left(x-\langle x\rangle_{l}\right) h(x, t)\right\rangle_{l},
$$

which is the slope of the straight line segment $\widetilde{h}_{l}(x, t)$ in the local window of size $l$ at a given time $t$ of a given interfacial configuration. Note that, throughout the paper, the term "local interfacial orientation" quantitatively refers to $s(l, t)$. Consequently, the original local width $w(l, t)$ and the modified local width $w_{n}(l, t)$ have the relation as follows:

$$
w^{2}(l, t)-w_{n}^{2}(l, t)=\frac{\left(l^{2}-1\right)}{12} \overline{\left\langle s^{2}(l, t)\right\rangle_{L}} .
$$

Here, $\overline{\left\langle s^{2}(l, t)\right\rangle_{L}}$ is obtained by first taking the average of

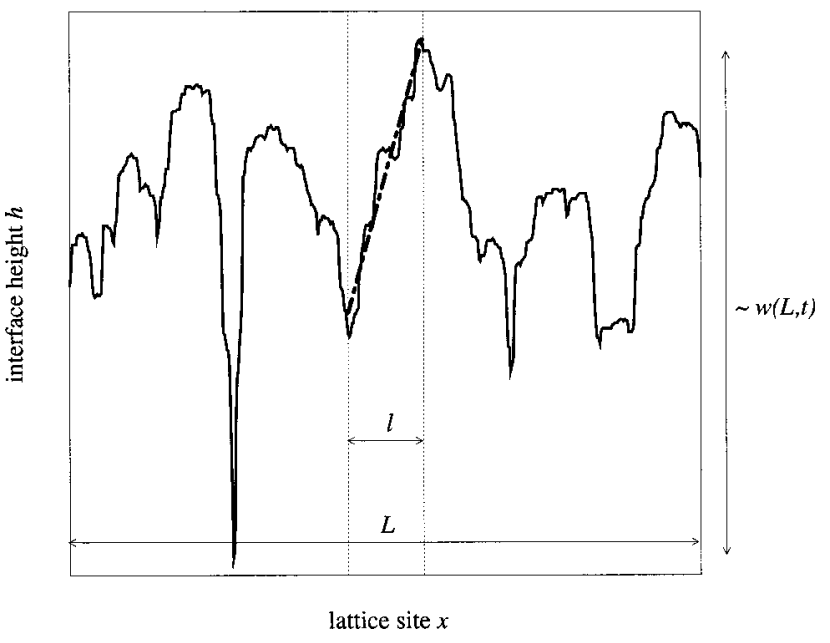

FIG. 1. A snapshot of typical $(1+1)$-dimensional superrough interface configuration in a system of size $L$. The solid curve represents the interface heights $h(x, t)$. The dot-dashed straight line segment represents $\widetilde{h}_{l}(x, t)$ obtained by least squares fit to the interfacial configuration within a local window of size $l(\ll$ the system size $L$ ).

$s^{2}(l, t)$ from many local windows of the same size $l$ along the $x$ axis over the whole system of size $L$ and then taking the statistical average. Figure 1 gives a pictorial explanation about the above related quantities.

Next, we want to first obtain the explicit relation between the correlation function, $G(r, t)$, and the average magnitude of the local interfacial orientation, ${\overline{\left\langle s^{2}(l, t)\right\rangle_{L}}}^{1 / 2}$. From Eq. (8),

$$
\begin{aligned}
& \overline{\left\langle s^{2}(l, t)\right\rangle_{L}}=\frac{144}{\left(l^{2}-1\right)^{2}} \overline{\left\langle\left\langle\left(x-\langle x\rangle_{l}\right) h(x, t)\right\rangle_{l}^{2}\right\rangle_{L}} \\
& =\frac{144}{\left(l^{2}-1\right)^{2}} \overline{\left\{\frac{1}{L} \sum_{x_{0}=1}^{L}\left[\frac{1}{l} \sum_{x^{\prime}=x_{0}}^{x_{0}+l-1}\left(x^{\prime}-x_{0}-\frac{l-1}{2}\right) h\left(x^{\prime}, t\right)\right]\left[\frac{1}{l} \sum_{x^{\prime \prime}=x_{0}}^{x_{0}+l-1}\left(x^{\prime \prime}-x_{0}-\frac{l-1}{2}\right) h\left(x^{\prime \prime}, t\right)\right]\right\}} \\
& =\frac{144}{\left(l^{2}-1\right)^{2} L l^{2}} \overline{\sum_{x_{0}=1}^{L} \sum_{x^{\prime}=x_{0}}^{x_{0}+l-1}\left(x^{\prime}-x_{0}-\frac{l-1}{2}\right)^{2} h\left(x^{\prime}, t\right)^{2}} \\
& +\frac{288}{\left(l^{2}-1\right)^{2} L l^{2}} \overline{\sum_{x_{0}=1}^{L} \sum_{x^{\prime}=x_{0}}^{x_{0}+l-2} \sum_{x^{\prime \prime}=x^{\prime}+1}^{x_{0}+l-1}\left(x^{\prime}-x_{0}-\frac{l-1}{2}\right)\left(x^{\prime \prime}-x_{0}-\frac{l-1}{2}\right) h\left(x^{\prime}, t\right) h\left(x^{\prime \prime}, t\right) .}
\end{aligned}
$$

By using the properties of periodic boundary conditions, we can easily obtain the first term, denoted by $A$, in the righthand side (rhs) of Eq. (10)

$$
A=\frac{12}{l\left(l^{2}-1\right)} \overline{\left\langle h\left(x^{\prime}, t\right)^{2}\right\rangle_{L}} .
$$

It demands much more effort to calculate the second term, denoted by $B$, in the rhs of Eq. (10). First, due to the translational invariance of the system, the spatial average and the statistical average are interchangeable. Then, by using the technique of change of variables, we can rewrite it as follows: 


$$
\begin{aligned}
B= & \frac{288}{\left(l^{2}-1\right)^{2} L l^{2}} \sum_{x_{0}=1}^{L} \sum_{x^{\prime}=x_{0}}^{x_{0}+l-2} \sum_{r=1}^{x_{0}+l-1-x^{\prime}}\left(x^{\prime}-x_{0}-\frac{l-1}{2}\right) \\
& \times\left(x^{\prime}+r-x_{0}-\frac{l-1}{2}\right) \overline{h\left(x^{\prime}, t\right) h\left(x^{\prime}+r, t\right) .}
\end{aligned}
$$

Moreover, the periodic boundary conditions give the following relation:

$$
\begin{aligned}
& \sum_{x_{0}=1}^{L} \sum_{x^{\prime}=x_{0}}^{x_{0}+l-2} \sum_{r=1}^{x_{0}+l-1-x^{\prime}} g\left(x^{\prime}, x_{0}\right) g\left(x^{\prime}+r, x_{0}\right) \\
& \quad=\sum_{x^{\prime}=1}^{L} \sum_{r=1}^{l-1} \sum_{x_{0}=x^{\prime}+r+1-l}^{x^{\prime}} g\left(x^{\prime}, x_{0}\right) g\left(x^{\prime}+r, x_{0}\right)
\end{aligned}
$$

for any function $g$. Thus, by employing Eq. (13), we obtain

$$
\begin{aligned}
& B=\frac{288}{\left(l^{2}-1\right)^{2} L l^{2}} \sum_{x^{\prime}=1}^{L} \sum_{r=1}^{l-1} \sum_{x_{0}=x^{\prime}+r+1-l}^{x^{\prime}}\left(x^{\prime}-x_{0}-\frac{l-1}{2}\right) \\
& \times\left(x^{\prime}+r-x_{0}-\frac{l-1}{2}\right) \overline{h\left(x^{\prime}, t\right) h\left(x^{\prime}+r, t\right)} \\
& =\frac{72}{\left(l^{2}-1\right)^{2} l^{2}} \sum_{r=1}^{l-1}(l-r) \\
& \times\left(\frac{(l-r)^{2}-1}{3}-r^{2}\right) \overline{\left\langle h\left(x^{\prime}, t\right) h\left(x^{\prime}+r, t\right)\right\rangle_{L}} .
\end{aligned}
$$

We then substitute Eq. (1) into Eq. (14) and obtain

$$
\begin{aligned}
B= & -\frac{12}{l\left(l^{2}-1\right)} \overline{\left\langle h\left(x^{\prime}, t\right)^{2}\right\rangle_{L}}+\frac{12}{l^{2}\left(l^{2}-1\right)} \sum_{r=1}^{l-1}(l-r) \\
& \times\left[\frac{2 r(r+l)}{\left(l^{2}-1\right)}-1\right] G(r, t) .
\end{aligned}
$$

From Eqs. (11) and (15), we thus obtain the relation between the average magnitude of the local interfacial orientation, ${\overline{\left\langle s^{2}(l, t)\right\rangle_{L}}}^{1 / 2}$, and the correlation function, $G(r, t)$, as follows:

$$
\overline{\left\langle s^{2}(l, t)\right\rangle_{L}}=\frac{12}{l^{2}\left(l^{2}-1\right)} \sum_{r=1}^{l-1}(l-r)\left[\frac{2 r(r+l)}{\left(l^{2}-1\right)}-1\right] G(r, t) .
$$

The local interfacial orientation, $s(l, t)$, definitely has close relations with any physical quantities related to interface slopes. For example, the step size (the nearest neighbor interface height difference) of the interface, $[G(1, t)]^{1 / 2}$, is exactly equal to ${\overline{\left\langle s^{2}(2, t)\right\rangle_{L}}}^{1 / 2}$. This equality can be easily obtained from Eq. (16). In addition, the slope-slope correlation function $\overline{\left\langle\nabla h\left(x_{0}, t\right) \nabla h\left(x_{0}+r, t\right)\right\rangle_{L}}$, proposed in Ref. [13], is also intimately related to $\overline{\left\langle s^{2}(l, t)\right\rangle_{L}}{ }^{1 / 2}$, which can be easily seen from Eqs. (1) and (16).

Subsequently, from Eqs. (1), (4), and (5), we can easily obtain the relation between the global interfacial width, $w(L, t)$, and the correlation function, $G(r, t)$, as

$$
w^{2}(L, t)=\frac{1}{2 L} \sum_{r=1}^{L-1} G(r, t)
$$

and the relation between the local interfacial width, $w(l, t)$, and the correlation function, $G(r, t)$, as

$$
w^{2}(l, t)=\frac{1}{l^{2}} \sum_{r=1}^{l-1}(l-r) G(r, t) .
$$

Then, by substituting Eqs. (16) and (18) into Eq. (9), we also obtain the expression of $w_{n}(l, t)$, the local interfacial width relative to the local interfacial orientation, in terms of the correlation function $G(r, t)$ as follows:

$$
w_{n}^{2}(l, t)=\frac{2}{l^{2}} \sum_{r=1}^{l-1}(l-r)\left[1-\frac{r(r+l)}{\left(l^{2}-1\right)}\right] G(r, t) .
$$

These relations, obtained above, are exact and thus can be applied to all the $(1+1)$-dimensional discrete growth processes with periodic boundary conditions, no matter whether the interface is superrough or not.

In the following, we want to employ the above obtained relations to study the asymptotic interfacial behaviors of the $(1+1)$-dimensional superrough growth processes in the intermediate and late time regimes. From Eqs. (2) and (3), we see that $G(r, t) \sim r^{2 \chi^{\prime}} t^{2 \kappa / z}$, with the local roughness exponent $\chi^{\prime} \equiv \chi-\kappa$, in the regime $r \ll t^{1 / z} \ll L$. On the other hand, $G(r, t) \sim r^{2 \chi^{\prime}} L^{2 \kappa}$ after the correlation length $\xi\left(\sim t^{1 / z}\right)$ reaches the system size $L$. Thus, the saturated global interfacial width $w_{\text {sat }}(L) \equiv w\left(L, t \gg L^{z}\right) \sim L^{\chi\left(=\chi^{\prime}+\kappa\right)}$. From a geometrical argument [14], it has been shown that the local roughness exponent $\chi^{\prime} \leqslant 1$. Consequently, for the systems with the correlation function $G(r, t)$ obeying the ordinary dynamic scaling ansatz, i.e., $\kappa=0$, the global roughness exponent $\chi$ must be smaller than or equal to 1 . Thus, the superrough growth processes (where $\chi>1$ ) must be accompanied by the anomalous dynamic scaling behaviors of $G(r, t)$. However, for those with $G(r, t)$ obeying the anomalous dynamic scaling ansatz, i.e., $\kappa \neq 0$, the global roughness exponent $\chi$ could be larger or smaller than one. Thus, we see that the anomalous dynamic scaling behaviors of $G(r, t)$ are necessary but not sufficient conditions for the superroughness of the interfaces. It has been known in the literature that the local interfacial width $w(l, t)$ of the superrough growth processes displays the same anomalous dynamic scaling behaviors as the correlation function $G(r, t)$. Namely, in the regime where the correlation length $\xi \sim t^{1 / z} \ll L, \quad w(l, t)$ $=l^{\chi} \widetilde{f}\left(l / t^{1 / z}\right)$ with the scaling function $\widetilde{f}(y) \sim y^{-\kappa}$ when $y$ $\ll 1$ and $\tilde{f}(y) \sim y^{-\chi}$ when $y \gg 1$. This result can also be easily seen from Eqs. (2), (3), and (18). Note that the nonsaturation of the scaling function $\widetilde{f}\left(l / t^{1 / z}\right)$, in the regime $l \ll t^{1 / z} \ll L$, gives rise to the substantial difference between global and local scaling behaviors. That is, the local interfacial width $w(l, t) \sim l^{\chi^{\prime}}$ at a fixed time slice $t$, in the regime $t^{1 / z} \gg l$; while the global interfacial width $w(L, t) \sim L^{\chi}$, in the regime $t^{1 / z}$ $\gg L$. Since the exponent $\kappa \neq 0$ in the superrough growth processes, the local roughness exponent $\chi^{\prime}$, which describes the spatial scaling behavior of the local interfacial width $w(l, t)$, 
has a different value from the global roughness exponent $\chi$, which describes the spatial scaling behavior of the global interfacial width $w(L, t)$. One has to take good care of this distinct feature, which is very crucial for correctly analyzing and interpreting the experimental data.

Next, we substitute $G(r, t) \sim r^{2 \chi^{\prime}} t^{2 \kappa / z}$ into Eq. (16) and obtain that the average magnitude of the local interfacial orientation has the anomalous temporal dependence, ${\overline{\left\langle s^{2}(l, t)\right\rangle_{L}}}^{1 / 2} \sim l^{\chi^{\prime}} t^{\kappa / z}$, in the regime $l \ll t^{1 / z} \ll L$. Note that, for the systems obeying the ordinary dynamic scaling ansatz, the local interfacial orientation saturates quickly after the growth time reaching the regime $t^{1 / z} \gg l$. Here, we explicitly verify, in $(1+1)$ dimensions, the usual conjecture [6] that all the superrough growth processes are associated with local interfacial orientational instability towards the creation of large slopes. Since all the superrough growth processes are associated with local interfacial orientational instability, it is interesting to find out whether local interfacial orientational instability alone causes the anomalous temporal dependence of the local interfacial width, $w(l, t) \sim l^{\chi^{\prime}} t^{\kappa / z}$, in the intermediate time regime $l \ll t^{1 / z} \ll L$. We then substitute the asymptotics of the correlation function $G(r, t) \sim r^{2 \chi^{\prime}} t^{2 \kappa / z}$, in the intermediate time regime $l \ll t^{1 / z} \ll L$, into Eq. (19) and obtain very intriguing results: (1) for the growth processes with $\chi^{\prime}<1$,

$$
\begin{aligned}
w_{n}^{2}(l, t) & \sim \frac{2}{l^{2}}\left\{\sum_{r=1}^{l-1}(l-r)\left[1-\frac{r(r+l)}{\left(l^{2}-1\right)}\right] r^{2} \chi^{\prime}\right\} t^{2 \kappa / z} \\
& \sim \frac{1-\chi^{\prime}}{\left(2 \chi^{\prime}+1\right)\left(\chi^{\prime}+1\right)\left(\chi^{\prime}+2\right)} l^{2 \chi^{\prime}} t^{2 \kappa / z},
\end{aligned}
$$

which indicates $w_{n}(l, t)$ retaining the same anomalous temporal dependence as the original local width $w(l, t)$; (2) while, for the growth processes with $\chi^{\prime}=1$,

$$
w_{n}^{2}(l, t) \sim \frac{2}{l^{2}}\left\{\sum_{r=1}^{l-1}(l-r)\left[1-\frac{r(r+l)}{\left(l^{2}-1\right)}\right] r^{2}\right\} t^{2 \kappa / z}=0,
$$

which indicates the leading asymptotic term of the correlation function $G(r, t), r^{2} t^{2 \kappa / z}$, having no contribution to the asymptotic behaviors of $w_{n}^{2}(l, t)$.

For the $(1+1)$-dimensional superrough growth processes with $\chi^{\prime}<1$, this robust anomalous temporal dependence of $w_{n}(l, t)$ implies that the local interface not only tilts, due to local interfacial orientational instability, but also forms local grooves or spikes as the time increases. Since the formation of local grooves or spikes is usually associated with the spatial multiscaling behaviors of interfaces, we thus conjecture that the interfaces of the $(1+1)$-dimensional superrough growth processes with the local roughness exponent $\chi^{\prime}<1$ display the spatial multiscaling behaviors. In contrast, for the $(1+1)$-dimensional superrough growth processes with $\chi^{\prime}$ $=1, w_{n}(l, t)$ either retrieves the ordinary dynamic scaling behaviors or has a much weakened temporal dependence (i.e., the lower order than $t^{\kappa / z}$ ) in the intermediate time regime $l \ll t^{1 / z} \ll L$. This result indicates that local interfacial orientational instability is the dominant mechanism causing the anomalous temporal dependence of $w(l, t)$ and, thus, the local interface of this class can be viewed as a normal selfaffine interface but gradually tilted as the time increases. We thus conjecture that the interfaces of the $(1+1)$-dimensional superrough growth processes with the local roughness exponent $\chi^{\prime}=1$ display spatial single scaling behaviors.

In conclusion, an extensive analytical study of the $(1+1)$-dimensional discrete superrough growth processes is undertaken. We obtain the exact expressions of the global interfacial width $w(L, t)$, the local interfacial width relative to the substrate orientation $w(l, t)$, and the local interfacial width relative to the local interfacial orientation $w_{n}(l, t)$, in terms of the equal-time height difference correlation functions $G(r, t)$. These relations are exact and can be applied to all the $(1+1)$-dimensional discrete growth processes with periodic boundary conditions, no matter whether the interface is superrough or not. Then we show that the anomalous dynamic scaling behaviors of $G(r, t)$ are necessary but not sufficient conditions for the superroughness (where the global roughness exponent $\chi>1$ ) of the interfaces. Moreover, we show that the local roughness exponent $\chi^{\prime}$ must be smaller than 1 for the $(1+1)$-dimensional superrough growth processes with $w_{n}(l, t)$ retaining the same anomalous dynamic scaling behaviors as $w(l, t)$; in contrast, the local roughness exponent $\chi^{\prime}$ must be equal to 1 for those with $w_{n}(l, t)$ retrieving the ordinary dynamic scaling behaviors. We then conjecture that the former class is associated with spatial multiscaling behaviors and the latter class is associated with spatial single scaling behaviors. It will be very interesting for future study to find out whether this conjecture is valid.

The work of N.-N. Pang was supported in part by the National Science Council of the Republic of China under Grant No. NSC 89-2112-M-002-012. The work of W.-J. Tzeng was supported in part by the National Science Council of the Republic of China under Grant No. NSC 89-2112-M032-016.
[1] Surface Disordering: Growth, Roughening, and Phase Transition, edited by R. Jullien, J. Kertesz, P. Meakin, and D. E. Wolf (Nova Science Publishers, Inc., Commack, 1992).

[2] T. Halpin-Healy and Y.-C. Zhang, Phys. Rep. 254, 215 (1995).

[3] P. Meakin, Fractals, Scaling \& Growth Far From Equilibrium (Cambridge University Press, Cambridge, 1998).

[4] M. Schroeder, M. Siegert, D. E. Wolf, J. D. Shore, and M.
Plischke, Europhys. Lett. 24, 563 (1993).

[5] J. Krug, Phys. Rev. Lett. 72, 2907 (1994).

[6] S. Das Sarma, C. J. Lanczycki, R. Kotlyar, and S. V. Ghaisas, Phys. Rev. E 53, 359 (1996).

[7] N.-N. Pang and W.-J. Tzeng, Phys. Rev. E 59, 234 (1999).

[8] H.-N. Yang, Y.-P. Zhao, G.-C. Wang, and T.-M. Lu, Phys. Rev. Lett. 76, 3774 (1996).

[9] J. H. Jeffries, J. K. Zuo, and M. M. Craig, Phys. Rev. Lett. 76, 
4931 (1996).

[10] S. Morel, J. Schmittbuhl, J. M. López, and G. Valentin, Phys. Rev. E 58, 6999 (1998).

[11] F. Family and T. Vicsek, J. Phys. A 18, L75 (1985).
[12] N.-N. Pang and W.-J. Tzeng, Phys. Rev. E 61, 3212 (2000).

[13] G. Palasantzas, Solid State Commun. 100, 699 (1996).

[14] H. Leschhorn and L.-H. Tang, Phys. Rev. Lett. 70, 2973 (1993). 